\title{
Vitamin D in the Clinic
}

\author{
Uriel S Barzel* \\ Professor Emeritus in Medicine, USA
}

Received: 制: November 19, 2018; Published: 制: November 26, 2018

*Corresponding author: Uriel S Barzel, Professor Emeritus in Medicine (Endocrinology), USA

Keywords: Vitamin D; Bone Disease; Deficiency; Parathyroid Glands; 25-hydroxyvitamin D; Hypercalcemia; Sarcoidosis

\section{Opinion}

The purpose of this short paper is to review clinical questions regarding vitamin D that arise in the course of treating patients with calcium problems or metabolic bone disease. These will include the optimal dosing of the vitamin, potential toxicities and impact on its use in the clinical setting. The information presented here is purely clinical, culled from years of clinical experience. Our patients in the Bronx have little or no exposure of their skin to sun light. This results in vitamin deficiency that sometime may be extreme. The reader will note that I make no reference to the medical literature, which is very extensive and grows daily. The first question is the dose of vitamin $\mathrm{D}$ to be used in any individual patient. To understand the reply, one must remember that the primary function of vitamin $\mathrm{D}$ is in the gut, facilitating the reabsorption of calcium from the internal recirculation and its absorption from dietary sources. These maintain blood calcium at a normal level. In the absence or insufficiency of vitamin D the blood level of calcium tends to fall. The parathyroid glands discharge some extra hormone, resulting in the resorption of bone and reabsorbing calcium by the kidneys.

The sensitivity of the parathyroid glands to fluctuations of calcium blood level is the key to the reply to the question of vitamin D dosing: If PTH (Para Thyroid Hormone blood level) is elevated and blood calcium is normal, one may suspect that vitamin D intake may be deficient. This, indeed, is true if the blood level of the metabolite - 25-hydroxyvitamin D - is below the normal range but also if it is in the normal range. The last statement is very significant:

a) The normal range is 30 to $100 \mathrm{ng} / \mathrm{ml}$. if a patient presents with a level of $32 \mathrm{ng} / \mathrm{ml}$, she may well be vitamin D deficient. One may be deficient even with levels of 40 or $60 \mathrm{ng} / \mathrm{ml}$ ! One's level is adequate only if PTH is normal.

b) Are there any physical signs that will suggest the need to evaluate these levels? There may be none. However, if a patient complains of bone pain and slight percussion of the clavicles elicits pain, the diagnosis of D deficiency is likely.
A case in point a woman in her fifties who was referred because she had a thyroid nodule. This was not a clinically significant problem, but the patient was very anxious, because she had bone pain. She had had surgery for breast cancer and was convinced that her disease had spread to her bones in spite of her oncologist reassurance, after extensive evaluation, that she is free of cancer. Her clavicles were extremely tender, her 25-hydroxyvitamin D blood level was below $10 \mathrm{ng} / \mathrm{ml}$ and her PTH markedly elevated. This leads me to the dosing of vitamin D deficiency: Patients require as much as 1 million international units for correction of severe deficiency. This woman was treated with 50,000IU biw for twelve weeks. When seen on follow-up she reported that the first two weeks had no clinical effect but on the third week of treatment she began to improve and after the fourth week she became convinced that she did not have metastatic bone disease. For maintenance, some patients require $1000 \mathrm{IU}$ daily, some $4000 \mathrm{IU}$ daily. This patient was advised to take $2000 \mathrm{IU}$ daily, for life, after reevaluation of blood levels of 25-hydroxyvitamin D and PTH.

Vitamin D toxicity results in hypercalcemia. This is an extremely rare complication. It is seen only in patients who consume hundreds of folds of the maintenance dose of the vitamin. Generally, this is a result of faulty industrial production. However, we have seen hypercalcemia in a patient who took 600,000IU daily, for over a week, from a foreign preparation said to be effective for chronic fatigue. Hypercalcemia in sarcoidosis is a unique problem. 25-hydroxyvitamin D blood level reflects the amount of the vitamin in storage. Its active metabolite, 1,25 dihydroxyvitamin D is produced by the kidneys under close control. In sarcoidosis, macrophages in the granulomas may have a 1-hydroxylase capacity that produces 1,25-dihydroxyvitamin D in an uncontrolled fashion. In this situation, 25-hydroxyvitamin $\mathrm{D}$ is consumed, such that the resulting hypercalcemia is associated, paradoxically, with a low blood level of 25-hydroxyvitamin D (and very low PTH).

A case in point is a young woman who was treated for vitamin D deficiency. She developed hypercalcemia that responded to saline 
administration, and discharged normocalcemic. Her physician checked 25 hydroxyvitamin D blood level, found it to be very low and prescribed vitamin D at a dose of 1000IU daily. Within 10 days, the patient had hypercalcemia again. At this point blood level of 25-hydroxyvitamin D was low but 1,25-dihydroxyvitamin D was elevated. Workup revealed the presence of sarcoidosis and the patient responded to steroid therapy. Osteoporosis presents a special challenge. Virtually all medications used in its treatment are "anti-resorptives". As described above, in the discussion of vitamin D dosing, the physiologic response to vitamin D deficiency is the stimulation of bone resorption. Thus, the use of anti-resorptives in persons with vitamin D deficiency, is physiologically inappropriate.
These persons must have their vitamin D status corrected prior to the administration of any bone-active medication.

In summary, the dosing of vitamin $\mathrm{D}$ depends on the degree of deficiency. Once corrected, patients must continue to take appropriate dose of vitamin D supplement for life. Hypercalcemia as toxicity of vitamin D administration is extremely rare, and results from ingesting extremely high doses of the vitamin. Hypercalcemia in sarcoidosis is the result of abnormal metabolism of the vitamin. Appropriate treatment of this condition may solve the calcium problem. Patients with osteoporosis should have normalization of vitamin D metabolism before anti-resoptives are prescribed.

\section{ISSN: 2574-1241}

DOI: 10.26717/BJSTR.2018.11.002084

Uriel S Barzel. Biomed J Sci \& Tech Res

CC (i) This work is licensed under Creative

Submission Link: https://biomedres.us/submit-manuscript.php

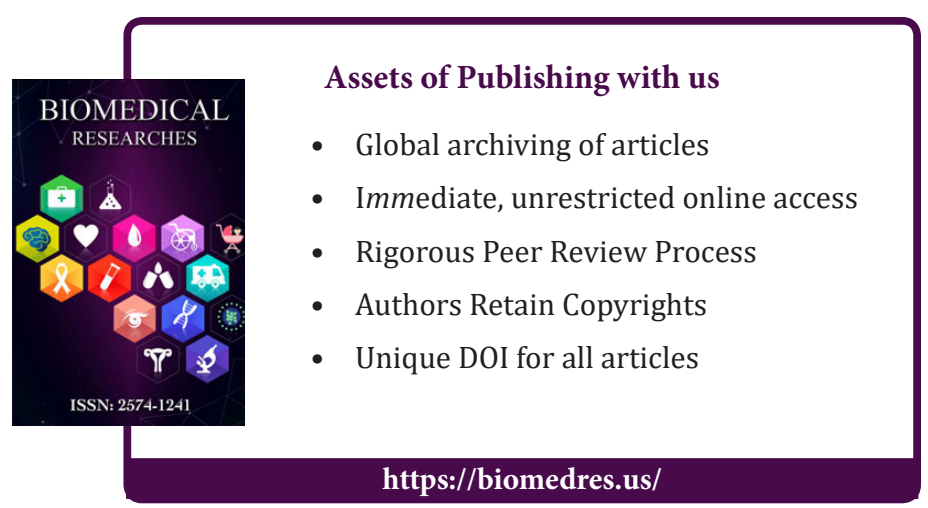

